$A(12 \cdot 14159$

\title{
AIAA 2002-0983
}

\section{A New Multifractal Subgrid Scale Model for Large Eddy Simulation}

Gregory C. Burton, Werner J.A. Dahm, Kenneth G. Powell and David R. Dowling

Laboratory for Turbulence \& Combustion (LTC)

Department of Aerospace Engineering

The University of Michigan

Ann Arbor, MI 48109-2140

Keck Laboratory for Computational Fluid Dynamics

Department of Aerospace Engineering

The University of Michigan

Ann Arbor, MI 48109-2140 


\title{
A New Multifractal Subgrid-Scale Model for Large-Eddy Simulation
}

\author{
Gregory C. Burton, ${ }^{*}$ Werner J.A. Dahm, ${ }^{\dagger}$ David R. Dowling, ${ }^{\ddagger}$ \\ and Kenneth G. Powell ${ }^{\S}$ \\ Laboratory for Turbulence and Combustion, and \\ W. M. Keck Foundation Laboratory for Computational Fluid Dynamics \\ Department of Aerospace Engineering \\ The University of Michigan, Ann Arbor, MI 48109
}

\begin{abstract}
We present a fundamentally new approach for the modeling of the subgrid-scale stresses for the large-eddy simulation (LES) of turbulent flows, based directly on the spatial distribution of vorticity within the subgrid field. Drawing on a substantial body of theoretical, experimental and computational evidence, we demonstrate that the enstrophy field $Q(\mathbf{x}, t) \equiv \frac{1}{2} \boldsymbol{\omega} \cdot \boldsymbol{\omega}(\mathbf{x}, t)$ exhibits multifractal scale-similarity in the inertial range of high Reynolds-number turbulence. A multifractal cascade can then be used to describe the spatial distribution of vorticity magnitudes within the subgrid field. An additive cascade can be used to describe the spatial distribution of vorticity orientations, which isotropically decorrelate through the subgrid field from the orientations of the smallest resolved scale $\Delta$ in the flow. It is then possible to recast the subgrid velocity contributions to the subgrid stress tensor $\tau_{i j}$ as Biot-Savart integrals over the subgrid vorticity field, which permits direct calculation of $\tau_{i j}$. The integral can be simplified using central-limit concepts, and in the high Reynolds-number limit, the subgrid-velocity field reduces to a simple algebraic expression based on quantities available from the resolved scales of the flow. Results from a priori tests are presented indicating good spatial and magnitude agreement for the filtered subgrid velocities $\overline{u^{s g s}}$, the subgrid stress tensor $\tau_{i j}^{*}$, and the subgrid energy production, $\mathcal{P}^{*}$. The multifractal approach presented here can be extended to model the filtered scalar transport equation and the Reynolds stresses in the Reynolds Averaged Navier-Stokes equation.
\end{abstract}

\section{Introduction.}

\section{A. SGS Modeling: Background.}

Given the enormous range of length and time scales present in high Reynolds-number turbulence, it is presently not feasible to calculate explicitly the entire range of features within turbulent flows. Instead, large-eddy simulation (LES) has been proposed as a means of dramatically reducing the computational expense of turbulent-flow simulations, by calculating exactly the large-scale features of turbulent flow, while modeling the small-scales. This approach has some intuitive appeal, since the large scales are presumably unique to each particular flow and are, thus, of greatest interest to the practicing engineer. By contrast, the small-scales of turbulent flows are presumably universal, and are significant only in their cumulative effect on the evolution of the large-scales. Thus, the development of an accurate model for the fine scales in turbulence holds the prospect of making LES a practical tool for real-

*FXB Fellow, Student Member AIAA, Corresponding Author.

†Professor, Associate Fellow AIAA.

$\ddagger$ Professor

$\S$ Professor, Member AIAA.

Copyright (C) 2002 by Gregory C. Burton. world engineering design and analysis.

All LES simulations require solving the filtered Navier-Stokes momentum equation,

$$
\frac{\partial \bar{u}_{i}}{\partial t}+\bar{u}_{j} \frac{\partial \bar{u}_{i}}{\partial x_{j}}-\nu \frac{\partial^{2} \bar{u}_{i}}{\partial x_{j}^{2}}+\frac{1}{\rho} \frac{\partial \bar{p}}{\partial x_{i}}=-\frac{\partial}{\partial x_{j}} \tau_{i j}
$$

where the overbar $(\because)$ represents the filtering operator, isolating the large scales in the flow. In general, the filtering operation can be expressed as a convolution of the given field with some filtering function $G(x)$ such that

$$
\bar{u}(\mathbf{x})=\int \boldsymbol{u}\left(\mathbf{x}^{\prime}\right) G\left(\mathbf{x}, \mathbf{x}^{\prime}\right) d \mathbf{x}^{\prime} .
$$

In an actual LES calculation, however, the filtered-velocity field $\bar{u}$ is a product of several factors, including the grid-spacing $\Delta$ and the discretization selected for the differential operators. The cumulative effect of these factors giving rise to the large-scale field is termed "implicit filtering". By contrast, in a priori testing of a subgrid model against a known DNS database, implicit filtering of the actual simulation can only be approximated by selection of an appropriate filter function $G(x)$ in 
(2), to isolate the large scales from the full-velocity field.

Whether explicit or implicit, the filtering operation over the non-linear advection term in the original Navier-Stokes momentum equation gives rise to the subgrid-stress tensor $\tau_{i j}$, defined as

$$
\tau_{i j}=\overline{u_{i} u_{j}}-\overline{u_{i}} \overline{u_{j}} .
$$

In this paper, the SGS stress tensor will be decomposed as

$$
\tau_{i j}=\frac{\overline{\overline{u_{i}}} \overline{\overline{u_{j}}}+\overline{\overline{u_{i}} u_{j}^{s g s}}+\overline{\overline{u_{i}^{s g s}} \overline{\overline{u_{j}}}}+}{}+
$$

indicating that the tensor contains contributions from both the resolved velocity $\bar{u}$ and subgrid velocity $u^{s g s}$. This decomposition is exact, and thus is equivalent mathematically to others appearing in the literature.

As the LES simulation explicitly calculates only the resolved-velocity field, determination of the subgrid stress tensor requires modeling the contributions of the subgrid velocity components, which appear in the decomposition in (4). These quantities are defined at scales between the LES-filter width $\Delta$ and the viscous-dissipation scale $\lambda_{\nu}$ within the subgrid field, and are not calculated explicitly by the simulation. This is the closure problem inherent in the LES formulation, and is the focus of all modeling efforts.

The cascade of kinetic-energy from large to smallscale features has been recognized as an important characteristic of turbulent flow for nearly 80 years, and significant effort has been devoted by the LES community to model properly the magnitude and spatial distribution of energy flux to the subgrid scales. The filtered kinetic-energy transport equation is given by

$$
\begin{array}{r}
\frac{D \bar{K}}{D t}-\frac{\partial}{\partial x_{j}}\left(2 \nu \bar{u}_{i} \bar{S}_{i j}-\frac{1}{\rho} \bar{u}_{i} \overline{p^{m}} \delta_{i j}-\bar{u}_{i} \tau_{i j}^{a}\right)= \\
-2 \nu \bar{S}_{i j} \bar{S}_{i j}-\mathcal{P} .
\end{array}
$$

where the subgrid-energy production term $\mathcal{P}$ is defined in terms of the resolved rate-of-strain tensor $\bar{S}_{i j}$ and the SGS stress tensor $\tau_{i j}$, as

$$
\mathcal{P}=-\tau_{i j} \bar{S}_{i j}
$$

In contrast to the classical view of the energytransfer process, which described a monotonic flux of energy from the large to small scales, recent studies have confirmed that turbulent flows routinely contain large regions of reverse energy transfer from the small to the large scales, termed backscatter. Explicit calculation of SGS energy production requires, therefore, accurate determination of the SGS stress tensor to account for the spatial variability in the magnitude and direction of SGS energy transfer.

\section{B. Early LES approaches.}

Large-eddy simulation has been an area of intense interest within the turbulence-modeling community for nearly forty years, and new models are proposed with some frequency. Most can be described broadly as either functional or structural models. As discussed at length in Sagaut (2000), functional models approximate the effect that the unresolved scales exert on the resolved field, most often by approximating the transport of energy between the resolved and subgrid fields. Structural modeling, by contrast, calculates the components of the subgrid stress tensor directly using information available from the filtered field.

In 1963, Smagorinsky first proposed the eddyviscosity formulation for the SGS stress tensor for the numerical modeling of turbulent atmospheric phenomena. A functional approach, the Smagorinsky model suggested that the SGS stress-tensor was proportional to the average resolved rate-of-strain $\bar{S}_{i j}$

$$
\tau_{i j}=-2 \nu_{t} \overline{S_{i j}}+\frac{1}{3} \tau_{k k} \delta_{i j},
$$

where the eddy-viscosity parameter $\nu_{t}$ could be modeled as

$$
\nu_{t}=C_{s} \Delta x^{2}|\bar{S}| .
$$

While loosely motivated by the gradient-transport hypothesis, Smagorinsky's formulation was essentially an ad hoc approach combined with dimensional reasoning to relate small-scale turbulence to the characteristics of the resolved flow. Since then, a number of modifications to the Smagorinsky model have appeared, most focusing on better estimating the eddy-viscosity parameter. Kraichnan (1976) proposed a spectral formulation in which Kolmogorov-type arguments were used to derive a value for the eddy-viscosity coefficient. The dynamic model of Germano et al (1991) retained the basic structure of the Smagorinsky model, but proposed a double-filtering operation on the resolved velocity field to permit local measurement of the eddy-viscosity parameter during the simulation.

The first significant structural approach reported in the literature was the scale-similarity model of Bardina et al (1983). The model calculated the SGS stress tensor directly, using a modified velocity field double-filtered near the smallest resolved scales in the flow, as

$$
\tau_{i j} \sim \overline{\overline{u_{i}}} \overline{\overline{u_{j}}}-\overline{\overline{u_{i}}} \overline{\overline{u_{j}}}
$$


While modeled SGS stresses correlated well with the actual flow, the model transferred insufficient energy from the resolved field, and Bardina proposed appending a linear eddy-viscosity term to the original model. Thus the final model combined both structural and functional elements, as

$$
\tau_{i j} \sim \overline{\overline{u_{i}} \overline{u_{j}}}-\overline{\overline{u_{i}}} \overline{\overline{u_{j}}}-2 C_{t} \Delta x^{2}|\bar{S}| \bar{S}_{i j} .
$$

Drawing on these previous efforts, Zang et al (1993) proposed local dynamic modification of the eddy-viscosity coefficient $C_{t}$, following the Germano approach, while retaining the form of Bardina's mixed scale-similarity model.

\section{Contemporary Approaches.}

Many recent LES models have significantly modified prior approaches, while others have explored fundamentally new techniques for subgridscale modeling. Recently proposed modifications to the eddy-viscosity approach include the structure function model of Metais \& Lesieur (1992). The approach modifies Kraichnan's (1976) spectral eddy viscosity formulation by use of a secondorder structure function, permitting local adjustment of the eddy-viscosity parameter, and thus better modeling of spatially-intermittent fields, such as subgrid-energy dissipation. New adaptive eddyviscosity/mixed approaches also have been explored by Ghosal et al (1995), who proposed a dynamiclocalization procedure, and Piomelli \& Liu (1995). In a related approach, Meneveau et al (1996) have proposed a Lagrangian method to locally calculate the eddy-viscosity parameter.

A number of notable structural models have been developed in recent years. For example, Domaradzki and colleagues have proposed a velocity-estimation technique for the calculation of the subgrid-stress tensor. First presented in a spectral form (Domaradzki \& Saiki 1997) and subsequently in a physical-space version (Domaradzki \& Loh 1999), the model proposes a two-step method, involving deconvolution and non-linear evolution, for approximating the velocity field at a scale one-half the size of the LES grid, which is then used to calculate the SGS tensor $\tau_{i j}$ directly. In a related approach, the approximate deconvolution procedure of Stoltz \& Adams (1998) employs an approximate inverse of the filtering operator to obtain a representation of the subgrid velocity field, which is then used to calculate the SGS tensor. Similarly, the inverse modeling approach of Guerts (1997) attempts to recover the subgrid velocity field from the filtered field with an approximate higher-order polynomial inversion of a spatial top-hat filter. Brassuer et al (1997) have formulated a new subgrid model in spectral space based on the dynamics of triadic interactions among local, non-local \& distant fourier modes. as a better means of capturing the transfer of energy into the subgrid field.

Several new approaches have incorporated vortex and vorticity dynamics directly into their SGS models. For example, Mansfield et al (1998) have proposed an LES scheme for the vorticity transport equation that draws on the Lagrangian eddyviscosity approach developed previously. In a related approach, Farge et al (1999) have proposed a coherent-vortex method that tracks the evolution of large vortices in the flow while modeling the distribution of smaller vortices. Drawing on the vortex tubes seen in DNS studies of high Reynolds-number turbulence, Pullin \& Saffman (1994) have proposed a vortex-structure model that approximates subgridscale turbulent structures as straight, rod-like cylinders of vorticity. From this, they derive a partly dynamic, partly stochastic representation of the SGS stress tensor. The model has been explored further by Misra \& Pullin (1997) who evaluated specific forms of the stochastic functions used in the vortexstructure model.

Branching out in a new direction is the fractalinterpolation method of Scotti \& Meneveau (1999), which constructs a synthetic subgrid velocity field using a fractal interpolation algorithm. The algorithm is an iterative mapping in one dimension that interpolates velocities between known points on a coarse mesh using fractal concepts.

\section{Overview of New Multifractal SGS Model.}

$\mathrm{By}$ contrast to these efforts, we present here a fundamentally new approach to the modeling of the subgrid-scale stresses, based directly on the spatial distribution of vorticity within the subgrid field. We are then able to recast the subgrid-velocity contributions to the subgrid-stress tensor in (4) as BiotSavart integrals over the subgrid vorticity field.

In order to derive a high-fidelity representation of subgrid vorticity, we draw on the substantial body of theoretical, experimental and computational evidence indicating that gradient quantities in turbulent flow are concentrated into multifractal structures in the inertial-range scales of high Reynoldsnumber turbulence. Multifractal concepts can then be used to describe an iterative cascade process that governs the spatial distribution of vorticity magnitudes within the subgrid field. We also describe an additive "orientation cascade" that governs the decorrelation of subgrid vorticity orientations from the orientation imposed by the smallest resolved scale $\Delta$ in the flow. Our approach therefore is to use the vorticity magnitude and orientation cascades to represent the spatial structure of the vorticity field within the subgrid field. This representation is then used in the Biot-Savart calculation to derive the 
components of the subgrid-velocity field $u_{i}^{\text {sgs }}$ that permits direct calculation of the SGS stress tensor $\tau_{i j}$.

The subgrid-scale model we describe below is fundamentally different from the approach of Scott \& Meneveau (1999) briefly mentioned above. That work involved a largely ad hoc effort to reconstruct the subgrid-velocity field directly from the resolvedvelocity field, based on fractal concepts and various interpolation techniques. However, no evidence supported the underlying assumption of that work that the subgrid-velocity field exhibits fractal or multifractal character. The present work, by contrast, draws on the known multifractal character of gradient-field quantities in high Reynolds-number turbulence to derive a model for the subgrid-velocity field, and therefore involves comparatively less of the ad hoc approaches present in most other SGS models.

\section{E. Paper Organization.}

This paper is organized into the following sections: Section 2 reviews multifractal concepts in general and methods for evaluating multifractal structure in a given field. Section 3 evaluates the multifractal structure in high Reynolds-number turbulence using high-resolution DNS data of homogeneous; isotropic turbulent flow. This analysis confirms that both the enstrophy and kinetic-energy dissipation fields exhibit multifractal scale-similar structure in the inertial range of high Reynolds-number turbulence. In section 4, the derivation of the present multifractal subgrid-scale model is set forth in some detail. It discusses (i) the spatial distribution of subgrid-vorticity magnitudes and orientations, (ii) the representation of the subgrid-velocity field using Biot-Savart integrals over the subgrid-vorticity field, and, (iii) the reduction of those integrals using central-limit concepts, from which the final form of the model emerges. Section 5 briefly discusses implementation of the model. Section 6 reports the results of a priori comparisons between the model and DNS data for three related field quantities: the filtered subgrid velocities, the SGS stress tensor components and the SGS energy production, and indicates that the model achieves good magnitude and phase agreement with DNS values. Section 7 extends the multifractal approach outlined in Sections $3 \& 4$ to a new subgrid-scale model for the filtered scalar-transport equation. The concluding remarks outline a multifractal model for the Reynolds Averaged Navier-Stokes equation.

\section{Review of Multifractal Concepts.}

Multifractal fields result naturally from the repeated application of a scale-invariant multiplicative process to an initial field. Detailed treatments of the multifractal formalism are given by Falconer (1990)

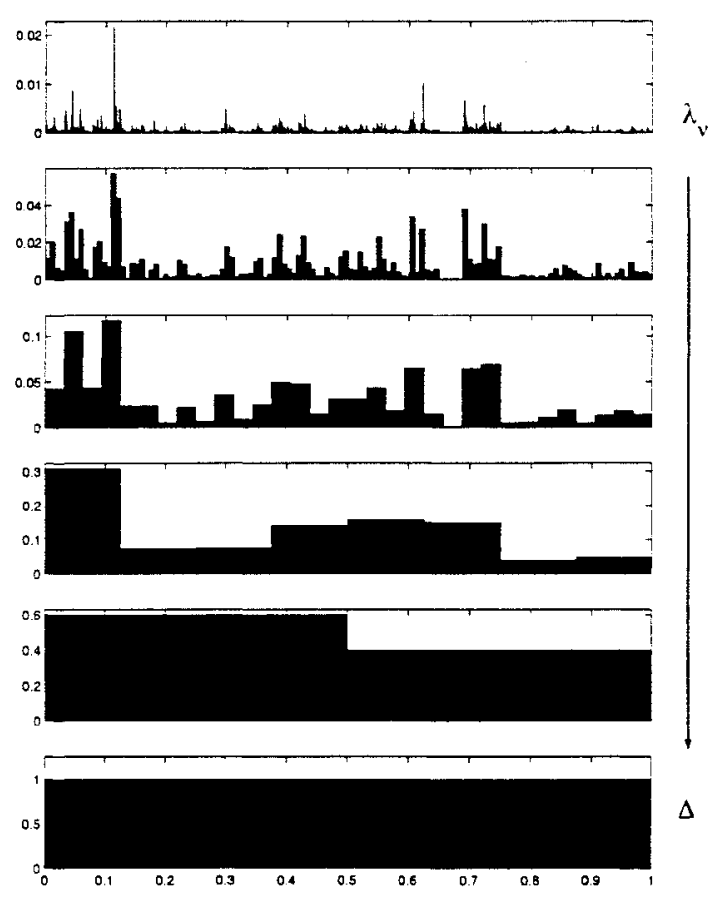

Fig. 1 Stochastic Cascade illustrated at five scales from $\lambda_{\nu} \rightarrow \Delta$. The cascade is a mass concentration process, by which some field, $\mu(x, t)$, is concentrated onto finer and finer subregions of the domain. The quantity of $\mu(x, t)$ distributed in each subregion at each scale is determined by the distribution of multipliers $P\left(\mathcal{M}_{\varepsilon}\right)$ illustrated in Figure 2.

and Pietgen et al (1992). In turbulent flows, such a multiplicative process is produced by the continual stretching and folding action of the time-varying strain rate and vorticity fields, with the required scale-invariance being naturally satisfied for scales sufficiently removed from the boundaries. Such considerations suggest that gradient quantities in turbulent flows, such as the enstrophy field $\frac{1}{2} \omega \cdot \omega$, the kinetic energy dissipation-rate field $-2 \nu \epsilon: \epsilon$, and the scalar energy dissipation rate field $-D \nabla \zeta \cdot \nabla \zeta$, will display multifractal scale-similarity. Indeed, early experimental investigations by Meneveau \& Sreenivasan $(1987,1991)$ concluded that the turbulent energy dissipation field exhibited multifractal characteristics. Meneveau (1991) subsequently used wavelet analyses to investigate scale similarity in the dissipation field, and found results consistent with multifractal scaling. Sreenivasan \& Stolovitzky (1995) examined scale similarity in detail and suggested the possibility of correlations in the cascade process. Similarly, experimental investigations by Prasad et al (1998), Sreenivasan \& Prasad (1989) and Frederiksen et al $(1996,1997)$ have confirmed that the scalar energy dissipation rate field in turbulent flows also displays multifractal scale similarity.

The simplest of such scale-invariant multiplica- 

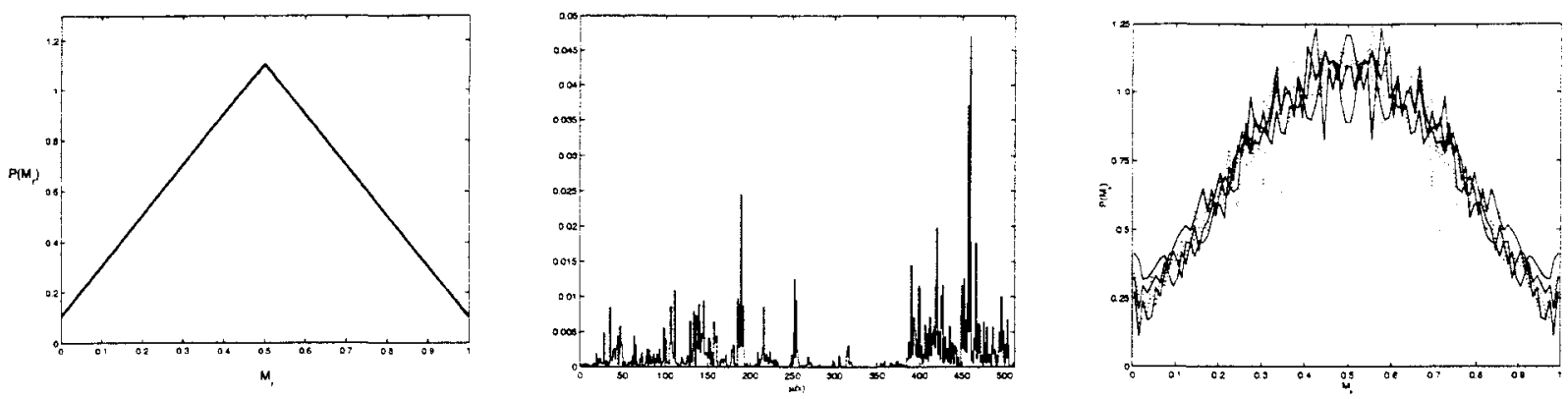

Fig. 2 Illustration of the multifractal cascade construction process. An invariant distribution of multipliers $P(\mathcal{M})$ (left) can be used to construct a stochastic realization of a multifractal test field of a given quantity $\mu(x)$ (center). The process can be inverted by using (11) to recover the original distribution (right).

tive processes are multiplicative cascades, in which a fixed set of multipliers $\mathcal{M}$ maps the field from one iteration to the next. In the deterministic binomial cascade, for example, at each stage of the construction, the "mass" contained in any given cell is distributed over two cells, each half the size of the previous stage, but with the mass multiplier $\mathcal{M}$ for each division now determined randomly from a scale-invariant distribution $P(\mathcal{M})$. After sufficiently many such repetitions, the resulting mass field $\mu(x)$ becomes highly intermittent. Owing to the stochastic nature of the generation process, arbitrarily many different realizations $\mu(x)$ can be produced from the same $P(\mathcal{M})$. Each such $\mu(x)$ differs in detail, but has the same underlying multiplicative scalesimilarity properties. Moments of the resulting fields are thus determined entirely by the underlying multiplier distribution $P(\mathcal{M})$.

A similar process can be applied "in reverse" to test for multifractal scale-similarity in a given field $\mu(x)$, based on the scale invariance of the multiplier distribution $P(\mathcal{M})$, as proposed by Sreenivasan (1991b) and Chhabra \& Sreenivasan (1992). The field $\mu(x)$ is used to obtain a $P\left(\mathcal{M}_{\varepsilon}\right)$ at each scale $\varepsilon$ by effectively reversing the multiplicative process. The multiplier $\mathcal{M}_{\varepsilon}$ between successive scales at any point $\left(\mathbf{x}_{i}, t_{i}\right)$ is thus determined as

$$
M_{\varepsilon}\left(\mathbf{x}_{i}, t_{i}\right) \equiv \frac{\int_{\left|\mathbf{x}-\mathbf{x}_{i}\right|<\varepsilon / \alpha} \int_{\left|t-t_{i}\right|<\varepsilon / \alpha} \mu(\mathbf{x}, t) d \mathbf{x} d t}{\int_{\left|\mathbf{x}-\mathbf{x}_{i}\right|<\varepsilon} \int_{\left|t-t_{i}\right|<\varepsilon} \mu(\mathbf{x}, t) d \mathbf{x} d t} .
$$

If the field is multifractal, then the resulting $P\left(\mathcal{M}_{\varepsilon}\right)$ at the various scales $\varepsilon$ will be scale-invariant. Frederiksen et al $(1996,1997)$ have used this process to establish that gradient fields in turbulent flows display multifractal scale similarity. The multiplier distribution $P(\mathcal{M})$ obtained from a multifractal field can then be used to generate synthetic fields which, over the range of scales at which the scale-invariant similarity applies, are statistically indistinguishable from the original field.

\section{The Multifractal Structure of Turbulent} Flow.

A substantial body of evidence, therefore, has been developed over the last decade and a half, based on theoretical, computational and experimental studies, indicating that gradient quantities in turbulent flow exhibit multifractal structure in the inertial-range of high Reynolds-number turbulence. No prior study, however, has directly addressed whether the enstrophy field, $Q(\mathbf{x}, t) \equiv \frac{1}{2} \omega \cdot \boldsymbol{\omega}(\mathbf{x}, t)$, exhibits such multifractal structure, although the same theoretical arguments must apply with equal force. We, therefore, obtained a high-resolution DNS database of turbulent flow in order to test for multifractal structure within the enstrophy field and to determine the distribution of multipliers $P\left(\mathcal{M}_{\varepsilon}\right)$ governing the spatial apportionment of subgrid enstrophy. The data consisted of a high-resolution $N=512^{3}$ simulation of forced, homogeneous, isotropic turbulence in a cubic, periodic domain, with Taylor-scale Reynolds number of $R e_{\lambda}=168$. The same data set was later employed to conduct the a priori studies summarized in Section 6. Further details concerning the DNS data can be found in Jimenez et al (1993).

As indicated in the typical one-dimensional extracts depicted in Figure 3, even at the moderate Reynolds number of the DNS data, both the enstrophy and kinetic-energy dissipation fields exhibit some of the spatial intermittency typical of high Reynolds-number turbulence, as these fields become concentrated onto smaller regions of the flow under the combined influence of the time-varying strain rate and vorticity fields. The multifractal character of both the enstrophy and the kinetic-energy dissipation fields were then evaluated using techniques outlined in Section 2. This consisted of comparing the ratio $\mathcal{M}_{\varepsilon}$ of each quantity at several pairs of adjoining scales within the flow, according to equation (11). The distribution of each ratio $P\left(\mathcal{M}_{\varepsilon}\right)$ was then evaluated for scale-invariance within the flow, which is the hallmark of multifractal spatial structure. Re- 

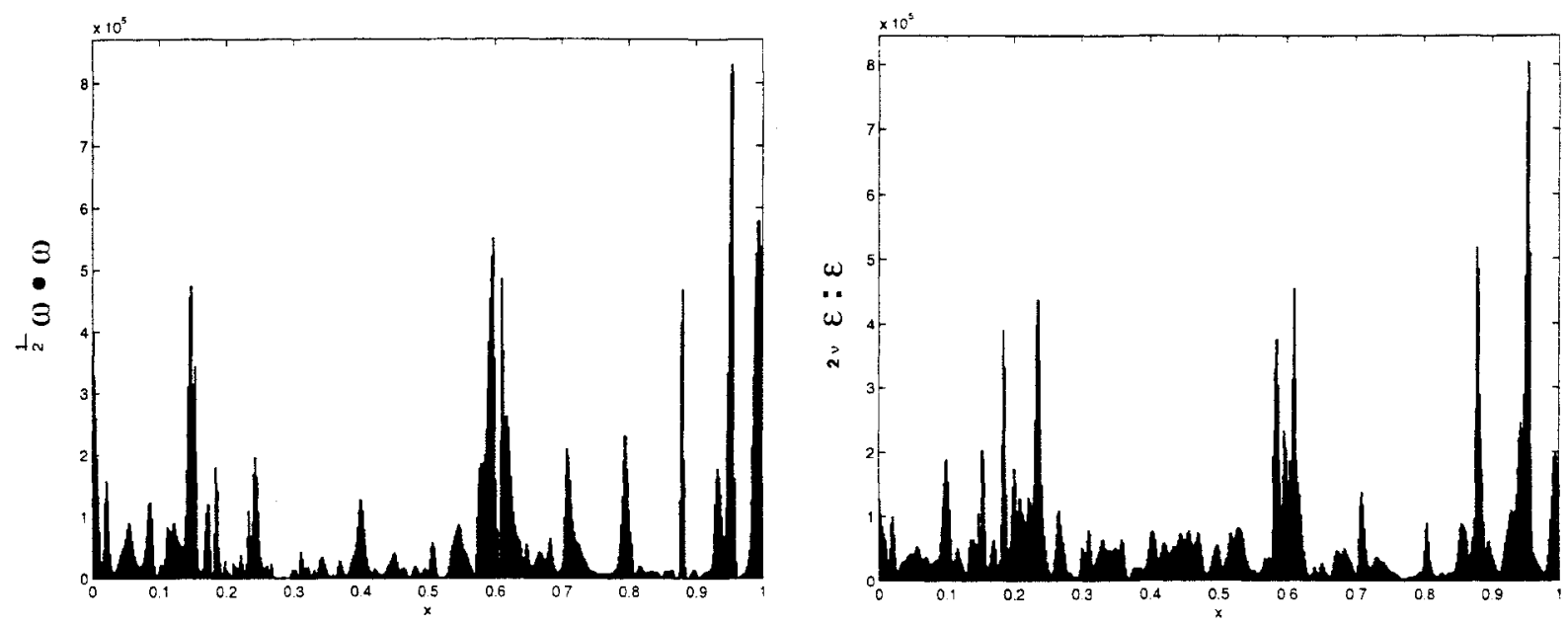

Fig. 3 One-dimensional extracts from enstrophy field (left) and kinetic-energy dissipation field (right) from homogeneous, isotropic DNS data, with $R e_{\lambda}=168$ and $N=512^{3}$, used to derive the distribution of multifractal multipliers illustrated in Figure 3.
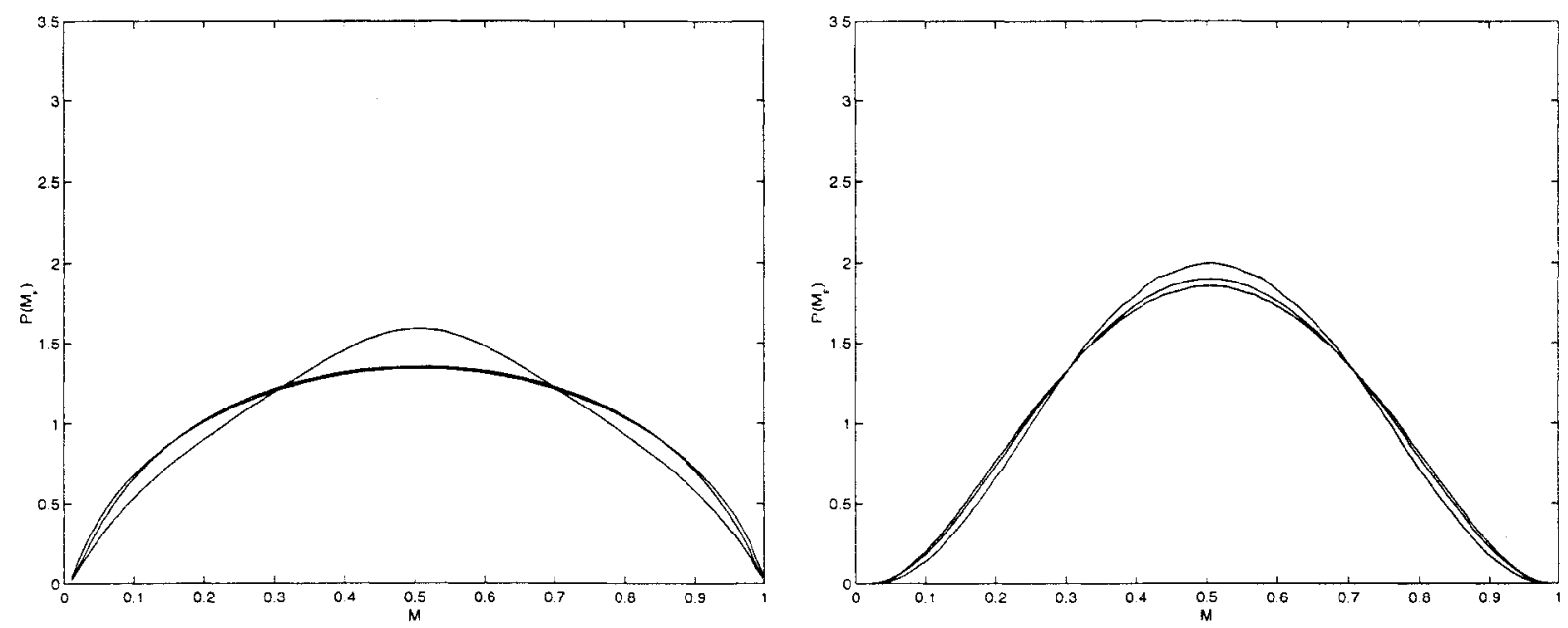

Fig. 4 Distributions of multifractal multipliers, $P\left(\mathcal{M}_{\varepsilon}\right)$ for the enstrophy field (left), and kineticenergy dissipation field (right) from DNS study of homogeneous, isotropic turbulence with $R e_{\lambda}=168$ and $N=512^{3}$. At this moderate Reynolds number, multifractal scale similarity is evident over three intermediate scales $\varepsilon$

sults of these evaluations are presented in Figures 4. These graphs confirm that both the enstrophy and the kinetic-energy dissipation fields exhibit multifractal scale-similarity over at least three scales within the particular DNS database evaluated. The multifractal structure apparent in the kinetic-energy dissipation field is consistent with the findings of earlier studies (Meneveau \& Sreenivasan 1991).

\section{Derivation of the Multifractal Subgrid-Scale Model.}

\section{A. Summary.}

The model presented here is based on a representation of the spatial distribution of subgrid vorticity within a single LES grid cell. Using the scaleinvariant multifractal multiplier distribution $P\left(\mathcal{M}_{\varepsilon}\right)$ for the enstrophy field depicted in Figure $4 a$, we first describe the spatial distribution of vorticity magnitudes within the subgrid field as a multiplicative cascade in accordance with basic multifractal principles. We then describe an additive orientation cascade governing the spatial distribution of subgrid vorticity orientations. In essence, the two cascades "fill out" vorticity magnitudes $\left|\boldsymbol{u}^{s g s}\right|$ and orientations $\hat{\mathbf{e}}^{s g s}$ within the subgrid field to the viscous scale $\lambda_{\nu}$, as depicted in Figure 5, using information from the smallest resolved scales $\Delta$ of the flow. These cascades remain sensitive to changes in the Reynolds number, by modifying the number of iterations as the ratio of LES to subgrid scales $\Delta / \lambda_{\nu}$ changes. Thus, as illustrated in Figure 6 , where the Reynolds number is smallest, (top), the viscous length-scale is largest and the number of iterations in the cascade remains small. However as the viscous 
$\Delta$

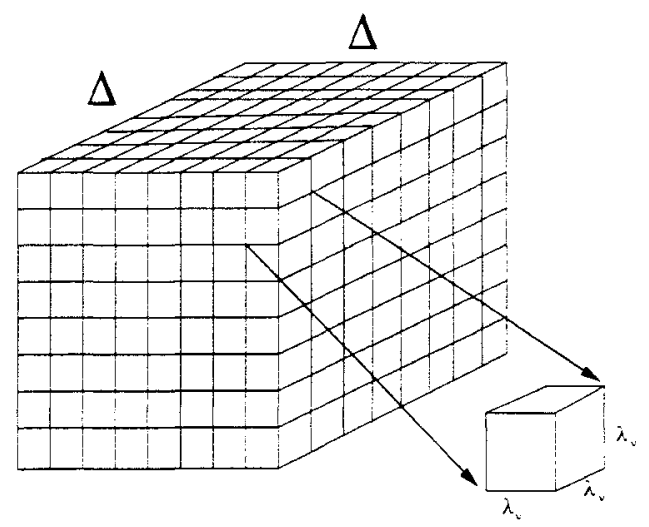

Fig. 5 Within a single LES grid-cell of scale $\Delta^{3}$, the described cascade processes distribute subgrid vorticity magnitudes $\left|\omega^{s g s}\right|$ and orientations $\hat{\mathrm{e}}^{s g s}$ at the viscous scale $\lambda_{\nu}$ within the subgrid field.

scale decreases with increasing Reynolds number, the number of cascade iterations increases (middle) and (bottom) resulting in the concentration of enstrophy onto smaller regions of the subgrid field.

With the spatial distribution of vorticity within the subgrid field thus described, the subgrid velocity $u_{i}^{s g s}$ can be represented as a Biot-Savart integral over the subgrid vorticity field, as

$$
u_{i}^{s g s}=\frac{1}{4 \pi} \int_{\Delta^{3}}\left(\omega^{s g s} \times \mathbf{K}\right) \cdot \hat{e}_{i} d^{3} \mathbf{x}^{\prime},
$$

where the traditional Biot-Savart kernel function $\mathbf{K}$ is defined as

$$
K \equiv \frac{x-x^{\prime}}{\left|x-x^{\prime}\right|^{3}}
$$

The subgrid velocity components in (12) are then inserted directly into the subgrid-stress tensor decomposition of equation (4), which closes the filtered LES momentum equation.

\section{B. Vorticity Magnitude Cascade.}

A complete description of the vorticity magnitude cascade requires determining both (i) the total amount of subgrid enstrophy and (ii) the rule governing its spatial distribution within the subgrid field. The total amount of subgrid enstrophy within a single LES grid-cell can be determined through a Kolmogorov spectral analysis. Recall that under K-41 theory, the enstrophy spectrum $Q(k)$ exhibits power law scaling in the inertial range of homogeneous, isotropic turbulence, as
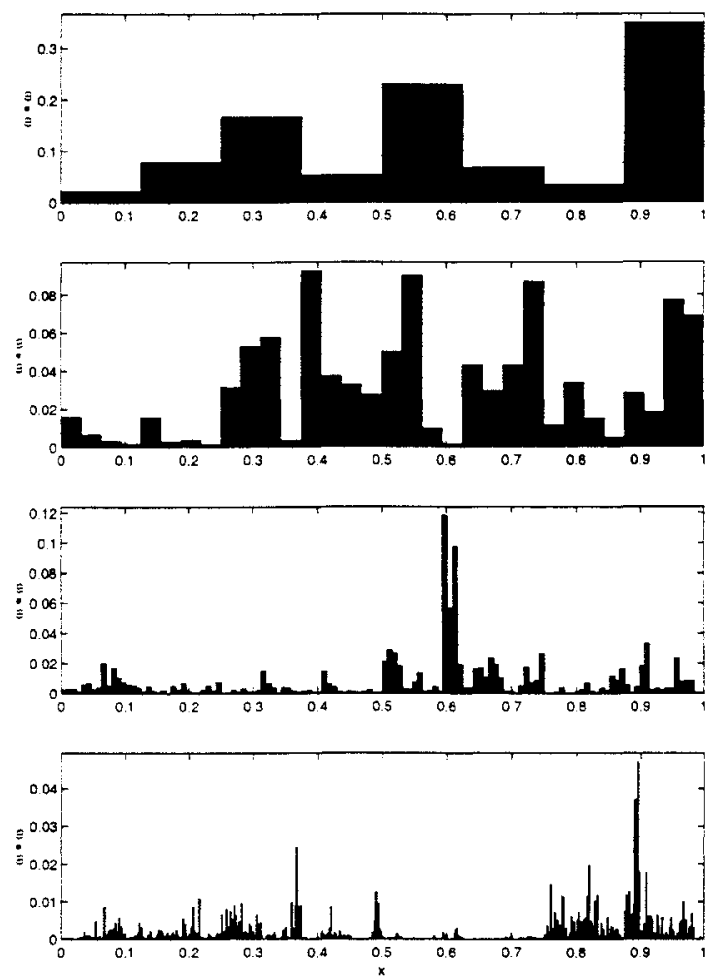

Fig. 6 Effect of increasing Reynolds number on cascade process. Four synthetic enstrophy fields at different Reynolds numbers, generated separately using the $P\left(\mathcal{M}_{\varepsilon}\right)$ of Figure 4 (left). At the smallest Reynolds number (top) the viscous scale $\lambda_{\nu}$ remains large and the cascade process contains few iterations. However, as the Reynolds number and the ratio $\frac{\Delta}{\lambda_{\nu}}$ increase, (middle) and (bottom), the number of cascade iterations increases, concentrating the enstrophy onto smaller regions of the subgrid field.

$$
Q(k) \sim k^{\frac{1}{3}} .
$$

As depicted in Figure 7, if the LES calculation explicitly resolves a portion of the inertial range, we can band-pass filter the resolved enstrophy field at the LES grid scale $\Delta$ to determine the amount of enstrophy $Q_{\Delta}$ that exists at that scale. Then using the power-law scaling, the enstrophy spectrum can be integrated from the smallest-resolved scale $k_{\Delta}$ to the viscous scale $k_{\nu}$ to determine the total amount of subgrid enstrophy $Q_{s g s}$, where

$$
\begin{aligned}
Q_{s g s} & =\int_{k_{\Delta}}^{k_{\nu}} Q(k) d k \\
& =\frac{3}{4} Q_{\Delta}\left[\left(\frac{k_{\nu}}{k_{\Delta}}\right)^{\frac{4}{3}}-1\right] .
\end{aligned}
$$

It is this quantity which is distributed spatially within the subgrid field according to the multifractal 


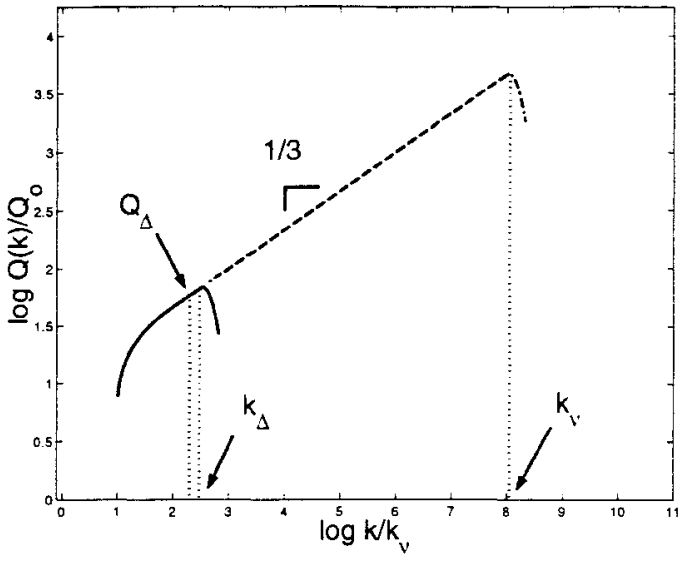

Fig. 7 Calculation of total enstrophy $Q_{s g s}$ within single LES grid-cell (idealized). If the LES calculation (solid) resolves into the inertial range, we can determine an amount of enstrophy at some small resolved scale $Q_{\Delta}$. Integration through the subgrid scales (dashed) gives the total amount of subgrid enstrophy that must be distributed according to the $P\left(\mathcal{M}_{\varepsilon}\right)$ distribution.

multiplier distribution $P\left(\mathcal{M}_{\varepsilon}\right)$. The current formulation of the model employs a single value for $\lambda_{\nu}$ throughout the computational domain. We are presently exploring methods for adaptively determining $\lambda_{\nu}$ based on fundamental dynamical concepts, which will permit more accurate determination of the total enstrophy in each LES grid-cell.

The final form for the rule governing the spatial distribution of this total subgrid enstrophy emerges directly from the multifractal principles explored in Section 2. Since the enstrophy cascade is an iterative, multiplicative process, it can be represented as a product of multiplicative factors $\mathcal{M}_{\varepsilon}$ drawn from the scale-invariant distribution of multipliers $P\left(\mathcal{M}_{\varepsilon}\right)$, represented in Figure 4, which concentrates the enstrophy onto smaller regions within the subgrid field. Thus, where $\Delta / \lambda_{\nu}$ represents the number of scales within the subgrid field, the expression

$$
\left|\omega^{s g s}(\mathbf{x}, t)\right|=\left[Q_{s g s} \prod_{i=1}^{\Delta / \lambda_{\nu}}\left(M_{\varepsilon}(\mathbf{x}, t)\right)_{i}\right]^{\frac{1}{2}},
$$

describes the spatial distribution of vorticity magnitudes throughout the subgrid scales.

\section{Vorticity Orientation Cascade.}

Significant experimental and computational evidence indicates that the orientations of the velocity field at the smallest resolved scale $u^{\Delta}$ in an LES calculation are correlated highly with the orientations of the true subgrid-velocity field. Liu \& Meneveau (1996) have shown that the orientations of a modified stress tensor $\tau_{i j}^{\Delta}$, calculated with a velocity field obtained from scales near the filter cut-off, are well

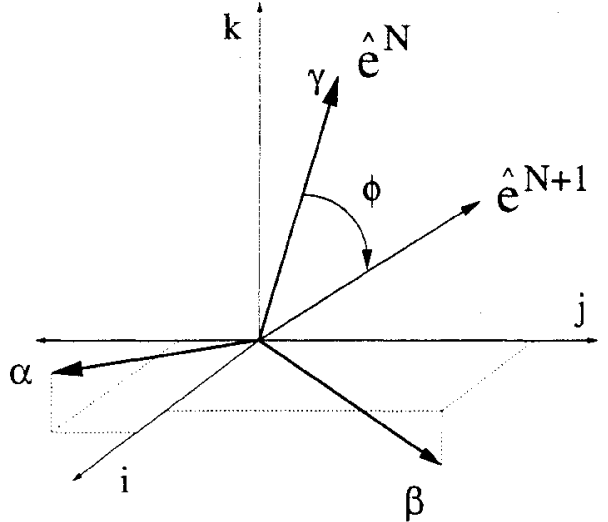

Fig. 8 The distribution of subgrid vorticity orientations is described by an "additive cascade" that decorrelates subgrid vorticity from the orientation of the smallest resolved scale $\Delta$. The particulars of the cascade are defined by the angle $\phi$ which quantifies the decorrelation of vorticity orientations at two adjoining scales, $\hat{\mathrm{e}}^{N}$ and $\hat{\mathbf{e}}^{N+1}$ within the subgrid field.

correlated, $\rho \sim 0.85$, with the orientations of the true subgrid scale stress tensor, in comparisons with experimental data from a high Reynolds-number jet. Similarly, the Bardina model produced high correlations $\rho \sim 0.8$ for $\tau_{i j}$ by using a velocity field also derived from scales near the grid cut-off. Most recently, Domaradzki's velocity-estimation approach reported high correlations for the SGS stress tensor calculated from a derived velocity field at a scale $\Delta / 2$.

Based on such work, the present subgrid-scale model takes the vorticity orientations of the smallest-resolved scale $\Delta$ as the departure point for describing an orientation cascade through the subgrid field. The cascade is taken to be an isotropic decorrelation from the orientations of the smallest resolved scales. Thus, at adjoining subgrid scales, the vorticity orientation at the smaller scale, $\hat{\mathbf{e}}^{N+1}$, deviates from the next larger scale, $\hat{\mathrm{e}}^{N}$, by a decorrelation angle $\phi$ as depicted in Figure 8 . The orientation at scale $N+1$ can be expressed as a sum of the orientation at scale $N$ and the decorrelation angle, $\phi$, as

$$
\hat{\mathbf{e}}_{i}^{N+1}=\hat{\mathbf{e}}_{i}^{N}+\phi_{i}
$$

The decorrelation process over all $\Delta / \lambda_{\nu}$ subgrid scales can then be expressed as a nested series of such quantities in (17), as 


$$
\hat{\mathbf{e}}_{i}^{s g s}(\mathbf{x}, t)=C_{\mathcal{M}}\left[\hat{\mathbf{e}}_{i}^{\Delta}(\mathbf{x}, t)+\delta_{i}^{s g s}(\mathbf{x}, t)\right]
$$

where

$$
\delta_{i}^{s g s}(\mathbf{x}, t) \equiv \sum_{n=1}^{\mathcal{M}}\left(\phi_{i}(\mathbf{x}, t)\right)_{n},
$$

and where $C_{\mathcal{M}}$ is an averaged normalization constant. The average value of the decorrelation sum

$$
\overline{\delta_{i}^{s g s}} \equiv 0
$$

since the orientation decorrelation is modeled as isotropic. Therefore the model properly recovers the orientation of the smallest resolved scale $\hat{\mathbf{e}}^{\Delta}$ when SGS vorticity orientations are filtered at the smallest-resolved scale $\Delta$, i.e.,

$$
\overline{\hat{e}^{s g s}}=\overline{\hat{e}^{\Delta}+\delta^{s g s}} \Rightarrow \hat{e}^{\Delta} .
$$

The multifractal magnitude cascade and the orientation cascade presented here are not entirely independent processes. Analysis of DNS data has established a direct relationship between the magnitude of the enstrophy multipliers $\mathcal{M}_{\varepsilon}$ and the correlation of vorticity orientations at adjoining scales within the subgrid field. Here we evaluated the distribution of decorrelation angles $P(\phi)$ associated with the enstrophy multiplier magnitudes. As illustrated in Figure 9, where enstrophy multipliers are smallest $\mathcal{M}_{\varepsilon}=0.02$ (top, left), the distribution of decorrelation angles is nearly uniform, indicating virtually no correlation between vorticity orientations at the two adjoining scales within the flow. However, as the enstrophy multiplier magnitude increases, a successively greater correlation between the adjoining scales is observed. At the largest value illustrated $\mathcal{M}_{\varepsilon}=0.95$ (bottom, right) the distribution approximates a delta function at $\phi=0$, indicating high correlation between the vorticity orientations at the adjoining scales. This behavior is consistent with the long-understood tendency of the strongest vortical structures to align preferentially with the principal extensional axis of the background strain field.

\section{Evaluation of the Biot-Savart Integrals.}

The multifractal magnitude cascade and the additive orientation cascade together provide a rigorous analytical representation for the spatial distribution of vorticity within the subgrid scales. Using the Biot-Savart operator, we now can derive an exact analytical expression for the subgrid velocity components $u_{i}^{s g s}$ appearing in the SGS stress tensor.

We first decompose the subgrid vorticity vector into magnitude and orientation portions, and apply the cascade representations to obtain the following expression for the subgrid vorticity field,
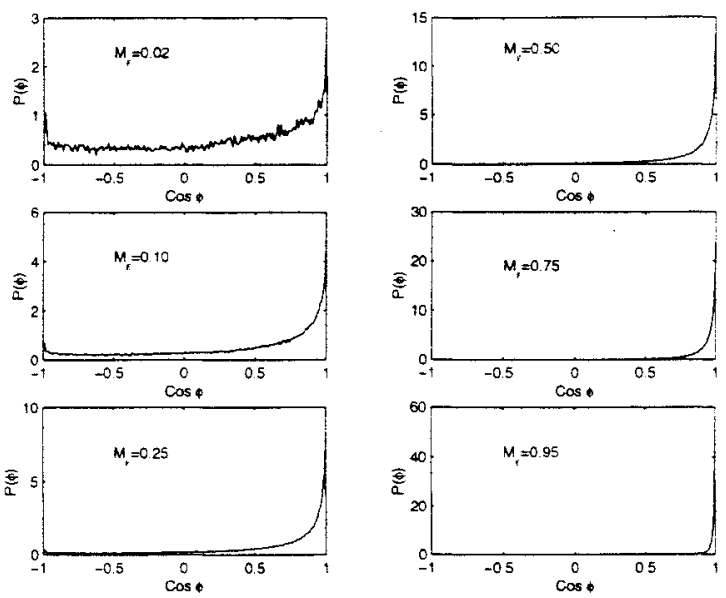

Fig. 9 Distribution of decorrelation angles $P(\phi)$ associated with six enstrophy multiplier values $\mathcal{M}_{\varepsilon}$ from DNS data. At the smallest value illustrated $\mathcal{M}_{\varepsilon}=0.02$ (top, left) the distribution of decorrelation angles in nearly uniform, while at the largest value illustrated $\mathcal{M}_{\varepsilon}=0.95$ (bottom, right) the distribution approximates a delta function at $\phi=0$, indicating little decorrelation. Thus, degree of correlation in vorticity orientation through the subgrid field is directly related to the magnitude of the associated multifractal multiplier $\mathcal{M}_{\varepsilon}$.

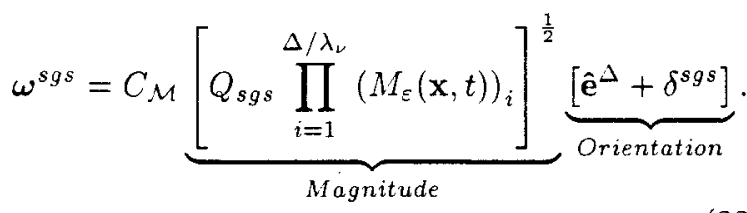

We then can obtain a representation for the subgrid velocity components $u_{i}^{s g s}$ using the Biot-Savart operator, as

$$
\begin{aligned}
& u_{i}^{s g s}=\underbrace{\frac{1}{4 \pi} \int_{\mathbf{x}^{\prime}}\left[\left(C_{\mathcal{M}}\left|\boldsymbol{\omega}^{s g s}\right| \hat{\mathbf{e}}^{\Delta}\right) \times \mathbf{K}\right] \cdot \hat{e}_{i} d^{3} \mathbf{x}^{\prime}}_{S^{\Delta}: \text { Resolved-Orientation }} \\
& +\underbrace{\frac{1}{4 \pi} \int_{\mathbf{x}^{\prime}}\left[\left(C_{\mathcal{M}}\left|\omega^{\text {sgs }}\right| \delta^{s g s}\right) \times \mathbf{K}\right] \cdot \hat{e}_{i} d^{3} \mathbf{x}^{\prime}}_{S^{\Phi}: \text { Subgrid Decorrelation }},
\end{aligned}
$$

where $\left|\omega^{s g s}\right|$ is substituted for the magnitude expression in (22).

The subgrid velocities are thus represented as the sum of two integrals, where

$$
u_{i}^{s g s}=\mathcal{S}^{\Delta}+\mathcal{S}^{\phi} .
$$

The resolved-orientation integral $\mathcal{S}^{\Delta}$ contains the contributions from the vorticity orientations within the subgrid field imposed by the smallest resolved scale $\Delta$. The subgrid-decorrelation integral $\mathcal{S}^{\phi}$ contains the contributions from the subgrid decorrelation cascade. 
The continuous integrals $\mathcal{S}^{\Delta}$ and $\mathcal{S}^{\varnothing}$ can be approximated by a discrete summation over all subgrid cells of volume $\lambda_{\nu}^{3}$, as

$$
\begin{aligned}
\mathcal{S}^{\Delta} & \approx \frac{C_{\mathcal{M}}}{4 \pi} \sum_{\text {all cells }}\left(|\omega|^{s g s} \hat{\mathrm{e}}_{j}^{\omega \Delta} K_{k}\right)_{\text {cell }} \lambda_{\nu}^{3} \\
\mathcal{S}^{\phi} & \approx \frac{C_{\mathcal{M}}}{4 \pi} \sum_{\text {all cells }}\left(|\boldsymbol{\omega}|^{s g s} \delta_{i}^{s g s} K_{k}\right)_{\text {cell }} \lambda_{\nu}^{3} .
\end{aligned}
$$

Each discrete summation contains stochastic quantities and may be analyzed using central-limit concepts. Thus, over many realizations, the value of each integral will assume a Gaussian probability distribution,

$$
\begin{aligned}
& \mathcal{S}^{\Delta} \approx P^{\Delta}\left(\frac{\mathcal{S}^{\Delta}-\overline{\mathcal{S}^{\Delta}}}{\sigma_{S^{\Delta}}} \leq z\right) \rightarrow \Phi(z) \\
& \mathcal{S}^{\phi} \approx P^{\phi}\left(\frac{\mathcal{S}^{\phi}-\overline{\mathcal{S}^{\phi}}}{\sigma_{S^{\phi}}} \leq z\right) \rightarrow \Phi(z),
\end{aligned}
$$

where $\overline{\mathcal{S}^{\Delta}}$ and $\sigma_{S^{\Delta}}^{2}$ represent the mean and variance of the resolved-orientation integral $\mathcal{S}^{\Delta}$, with similar notation for the subgrid-decorrelation integral $\mathcal{S}^{\phi}$. In this analysis, we have made the simplifying assumption that the multifractal multipliers do not induce correlations in the spatial distribution of subgrid vorticity magnitude.

Then, assuming constant values for the magnitudes of the resolved-velocity field $\left|\mathbf{u}^{\Delta}\right|$, the resolved vorticity field $\left|\omega^{\Delta}\right|$, and the subgrid vorticity field $\left|\omega^{s g s}\right|$ in each LES grid-cell, the mean for the resolved-orientation distribution $\overline{\mathcal{S}^{\Delta}}$ can be approximated as,

$$
\overline{\mathcal{S}^{\Delta}}=\left|\mathbf{u}^{\Delta}\right| \frac{\left|\boldsymbol{\omega}^{s g s}\right|}{\left|\boldsymbol{\omega}^{\Delta}\right|} \hat{\mathbf{e}}_{i}^{\mathbf{u}^{\Delta}} .
$$

A similar analysis can be applied to the decorrelation integral $\mathcal{S}^{\phi}$, noting that its mean $\overline{\mathcal{S}^{\phi}}$ is zero due to the isotropic nature of the decorrelation within the subgrid field. Thus, as depicted in Figure 10, the expression for the subgrid-velocity field $u_{i}^{s g s}$ simplifies to the sum of two Gaussian distributions,

$$
\begin{aligned}
u_{i}^{s g s}= & \underbrace{P^{\Delta}\left(\frac{\mathcal{S}^{\Delta}-\left|\mathbf{u}^{\Delta}\right| \frac{\left|\boldsymbol{\omega}^{s g}\right|}{\left|\boldsymbol{\omega}^{\Delta}\right|} \hat{\mathbf{e}}_{i}^{\mathbf{u}^{\Delta}}}{\sigma_{S^{\Delta}}} \leq z^{\Delta}\right)}_{\mathcal{S}^{\Delta}} \\
& +\underbrace{P^{\phi}\left(\frac{\mathcal{S}^{\phi}}{\sigma_{s^{\phi}}} \leq z^{\phi}\right)}_{\mathcal{S}^{\phi}},
\end{aligned}
$$

where $P^{\Delta}$ is derived from the resolved-orientation integral with non-zero mean, and $P^{\phi}$ from the

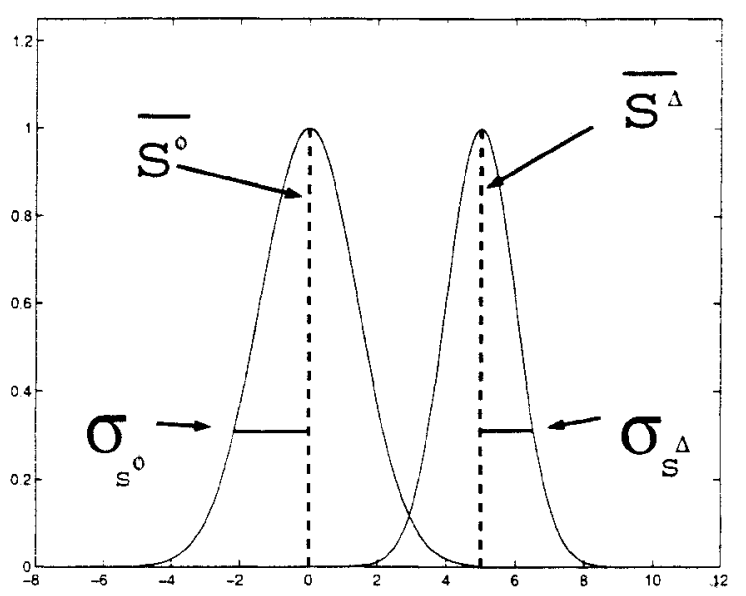

Fig. 10 Gaussian representation of the BiotSavart integrals (idealized). The central-limit theorem reduces the integral representation of the subgrid-velocity field to a pair of Gaussian distributions. The mean of the resolvedorientation distribution (right) is calculated as $\overline{\mathcal{S}^{\Delta}}=C(\mathbf{x}, t) \hat{\mathbf{e}}_{i}^{\mathbf{u}^{\Delta}}(\mathbf{x}, t)$, with $C(\mathbf{x}, t)$ defined in (35). By contrast, the mean of the subgriddecorrelation distribution $\overline{\mathcal{S}^{\phi}}$ equals zero since the decorrelation process is taken as isotropic through the subgrid field (left). In the high-Re limit, both variances asymptote to zero, leaving $u_{i}^{s g s}=C \hat{\mathrm{e}}_{i}^{\mathrm{u}^{\Delta}}$ as the final form for the multifractal representation of the subgrid velocity field.

subgrid-decorrelation integral with a zero mean value.

\section{E. High Reynolds-Number Limit Analysis.}

As the Reynolds number increases, the ratio of viscous to outer length scales in each LES grid-cell,

$$
\frac{\lambda_{\nu}}{\Delta} \rightarrow 0
$$

while the number of subgrid cells of volume $\lambda_{\nu}^{3}$ within each LES grid-cell

$$
N\left(\lambda_{\nu}^{3}\right) \rightarrow \infty
$$

and the variances of the two Gaussian distributions $\mathcal{P}^{\Delta}$ and $\mathcal{P}^{\phi}$

$$
\begin{aligned}
& \sigma_{s^{\Delta}}^{2} \rightarrow 0 \\
& \sigma_{s^{\Phi}}^{2} \rightarrow 0 .
\end{aligned}
$$

The probability distributions in (28) governing the value of the subgrid velocity field $\boldsymbol{u}^{\text {sgs }}$ therefore contract around their mean values:

$$
\mathcal{S}^{\Delta} \approx P\left(\frac{\mathcal{S}^{\Delta}-\overline{\mathcal{S}^{\Delta}}}{\sigma_{S^{\Delta}}} \leq z\right) \Rightarrow\left|\mathbf{u}^{\Delta}\right| \frac{\left|\omega^{s g s}\right|}{\left|\omega^{\Delta}\right|} \hat{\mathbf{e}}_{i}^{\mathbf{u}^{\Delta}}
$$




$$
\mathcal{S}^{\phi} \approx P^{\phi}\left(\frac{\mathcal{S}^{\phi}}{\sigma_{S^{\phi}}} \leq z^{\phi}\right) \Rightarrow 0 .
$$

Thus, in the high Reynolds-number limit, the subgrid velocity field $u_{i}^{s g s}=\mathcal{S}^{\Delta}+\mathcal{S}^{\phi}$ can be represented by the simple functional form,

$$
u_{i}^{s g s}(\mathbf{x}, t) \approx C(\mathbf{x}, t) \hat{\mathbf{e}}_{i}^{\mathbf{u}^{\Delta}}(\mathbf{x}, t),
$$

where

$$
C(\mathbf{x}, t) \equiv\left|\mathbf{u}^{\Delta}(\mathbf{x}, t)\right| \frac{\left|\boldsymbol{\omega}^{s g s}(\mathbf{x}, t)\right|}{\left|\boldsymbol{\omega}^{\Delta}(\mathbf{x}, t)\right|} .
$$

The subgrid velocity field $\boldsymbol{u}^{\text {sgs }}$ is now expressed solely in terms of quantities available from the resolved scales of the flow. The full SGS stress tensor $\tau_{i j}$ can be represented as

$$
\tau_{i j}=\frac{\overline{\overline{u_{i}} \overline{u_{j}}}+\overline{\overline{u_{i}} C \hat{\mathbf{e}}_{j}^{\overline{\mathbf{u}^{\Delta}}}}+\overline{C \hat{\mathbf{e}}_{i}^{\mathbf{u}^{\Delta}} \overline{\overline{u_{j}}}}+}{C \overline{\hat{\mathbf{e}}_{i}^{\mathbf{u}^{\Delta}} C \hat{\mathbf{e}}_{j}^{\mathbf{u}^{\Delta}}}-\overline{u_{i}} \overline{u_{j}}}
$$

which is the final form of the present multifractal subgrid-scale model.

\section{Implementation of Multifractal Subgrid-Scale Model.}

The subgrid-scale modeling approach presented here involves explicit calculation of subgrid velocity components $u_{i}^{\text {sgs }}$ appearing within the decomposition of the subgrid-stress tensor. The model expresses these subgrid components as functions of quantities at the smallest-resolved scale $\Delta$ in the LES simulation, i.e.,

$$
u_{i}^{s g s} \equiv \mathcal{F}\left(\left|\mathbf{u}^{\Delta}\right|,\left|\boldsymbol{\omega}^{\Delta}\right|,\left|\boldsymbol{\omega}^{s g s}\right|, \hat{\mathbf{e}}_{i}^{\mathbf{u}^{\Delta}}\right) \equiv \mathcal{F}\left(C, \hat{\mathbf{e}}_{i}^{\mathbf{u}^{\Delta}}\right),
$$

where $C$ is defined by equation (35). Each of these quantities represents a continuous field which is sampled on the LES grid. These fields, in turn, appear under a filtering operator in the expression for the SGS stress tensor, which here is taken to represent a three-dimensional spatial average over a single LES grid-cell of size $\Delta^{3}$. Thus, calculation of each term of the decomposed SGS stress tensor, e.g., $\overline{u_{i}^{s g s} \overline{u_{j}}}$, is represented using the multifractal model as

$$
\overline{u_{i}^{s g s} \overline{u_{j}}}=\overline{C \hat{\mathbf{e}}_{i}^{\mathbf{u}^{\Delta}} \overline{u_{j}}}=\frac{1}{\Delta^{3}} \int\left(C \hat{\mathbf{e}}_{i}^{\mathbf{u}^{\Delta}} \overline{u_{j}}\right) d^{3} \mathbf{x}^{\prime} .
$$

Explicit calculation of $(38)$ requires selecting a particular mathematical form for the quantities appearing in the integrand. Because these fields are continuous quantities, they contain variations within each grid-cell $\Delta^{3}$ in which the spatial averaging occurs. Thus, each field can be expressed within an LES grid-cell as

$$
\begin{aligned}
\overline{u_{i}}\left(\mathbf{x}, t_{o}\right) & =\overline{u_{i}}\left(x_{o}, t_{o}\right)+\nabla \overline{u_{i}}\left(\mathbf{x}, t_{o}\right) \cdot \mathbf{r} \\
\hat{\mathbf{e}}_{j}^{u^{\Delta}}\left(\mathbf{x}, t_{o}\right) & =\hat{\mathbf{e}}_{j}^{u^{\Delta}}\left(x_{o}, t_{o}\right)+\nabla \hat{\mathbf{e}}_{j}^{u^{\Delta}}\left(\mathbf{x}, t_{o}\right) \cdot \mathbf{r} \\
C\left(\mathbf{x}, t_{o}\right) & =C\left(x_{o}, t_{o}\right)+\nabla C\left(\mathbf{x}, t_{o}\right) \cdot \mathbf{r},
\end{aligned}
$$

where $t_{o}$ represents the current time-step and $x_{0}$ the location of the LES grid-cell centers. Thus, a filtered component of the subgrid stress tensor in (36) can be evaluated as

$$
\begin{aligned}
\overline{C \hat{\mathbf{e}}_{i}^{u^{\Delta}} \overline{u_{j}}} & =\frac{1}{\Delta^{3}} \int_{\Delta^{3}}\left[C\left(x_{o}, t_{o}\right)+\nabla C\left(\mathbf{x}, t_{o}\right) \cdot \mathbf{r}\right] \times \\
& {\left[\hat{\mathbf{e}}_{i}^{u^{\Delta}}\left(x_{o}, t_{o}\right)+\nabla \hat{\mathbf{e}}_{i}^{u^{\Delta}}\left(\mathbf{x}, t_{o}\right) \cdot \mathbf{r}\right] \times } \\
& {\left[\overline{u_{j}}\left(x_{o}, t_{o}\right)+\nabla \overline{u_{j}}\left(\mathbf{x}, t_{o}\right)\right] d^{3} \mathbf{x}^{\prime} . }
\end{aligned}
$$

In the present work, we explicitly calculate the stress tensor terms using a quadratic representation of all resolved field quantities. The gradients in the representation are determined by a unweighted least-squares approximation based on the twenty-six nearest neighbors of the individual LES grid-cell. Higher-fidelity interpolation procedures are currently being explored, where least-squares weighting schemes are based on an approximate inversion of the spatial-filtering operator.

\section{Multifractal Subgrid-Scale Model Validation.}

We have conducted an initial series of a priori tests to assess the validity of the multifractal model, as well as those assumptions underlying its derivation. These tests employed the same DNS data used for the multifractal analysis discussed in Section 3. We specifically examined the model's ability to recover three relevant characteristics of the LES flow: (i) the filtered subgrid-velocity components $\overline{u_{i}^{s g s}}$, (ii) the components of the subgrid-stress tensor $\tau_{i j}^{*}$, and, (iii) the SGS-energy production field $\mathcal{P}^{*} \equiv-\tau_{i j}^{*} \bar{S}_{i j}$.

The DNS values for comparison were derived by calculating each quantity at the resolution of the original DNS study, here $N=512^{3}$, and then spatially averaging the given quantity over a single LES grid cell, using a traditional three-dimensional boxcar filter. Filter width $\Delta$ was selected at

$$
\Delta=16 d x
$$

where $d x \equiv L / 512$ and $L$ the outer-length scale of the computational domain. The selected filter width ensured that the subgrid enstrophy field contained scales where multifractal structure had been confirmed.

We first examined the model's ability to recover the filtered components of the subgrid-velocity field 
$\overline{u_{i}^{s g s}}$. The quantity was selected as perhaps the most direct means of assessing the accuracy of the model. Figure 11 sets forth six random one-dimensional intersections from the filtered subgrid-velocity field, comparing DNS values (solid) and model values (dashed). Global correlations between the two fields were calculated to be $\rho \sim 0.91$. Each of the intersections shows good magnitude as well as spatial agreement, indicating that the model is able to recover significant structural characteristics of the field.

We next compared DNS and model values of the subgrid stress tensor, $\tau_{i j}$. This is, of course, the central focus of all structural LES modeling efforts, since proper representation of the subgrid-stress tensor would close the filtered Navier-Stokes momentum equation. Since the model presented here explicitly represents the subgrid-velocity field, we focused our comparison on that portion of the SGS stress tensor $\tau_{i j}^{*}$, containing all the contributions from the subgrid-velocity field, where

$$
\tau_{i j}^{*} \equiv \overline{\overline{u_{i}}} u_{j}^{s g s}+\overline{u_{i}^{s g s} \overline{u_{j}}}+\overline{u_{i}^{s g s} u_{j}^{s g s}} .
$$

This comparison was selected as the best means of evaluating the contributions of the model to the value of the stress tensor. By contrast, the remaining stress-tensor terms in (4), $\overline{\overline{u_{i}} \overline{u_{j}}}$ and $\overline{u_{i}} \overline{u_{j}}$, concern the resolved scales of the flow exclusively and are unrelated to the subgrid model proposed here. Global correlations between the actual and modeled SGS stress tensor component field were calculated as $\rho \sim 0.855$. A number of one-dimensional intersections depicted in Figure 12 were extracted from the original three-dimensional fields, to visually assess the model's ability to capture significant features of the actual stress-tensor field. DNS values (solid) are shown against the modeled subgrid values (dashed). Examination of these fields indicates that the model recovers much of the important spatial distribution of tensor component orientations and magnitudes.

The final a priori test compared the modeled subgrid energy-production field with the DNS data. This is often considered the litmus test of a subgrid model, since properly recovering the spatial distribution of subgrid energy transfer is thought to be essential for accurately calculating the large-scale structures in the flow. In order to focus exclusively on the performance of our subgrid model, the comparison again involved only that portion of the SGS energy production field $\mathcal{P}^{*}$ due directly to the contribution of the subgrid-velocity terms, where

$$
\mathcal{P}^{*} \equiv-\tau_{i j}^{*} \bar{S}_{i j}
$$

where $\tau_{i j}^{*}$ is defined in (42).

Since subgrid energy production is dependent on the phase and magnitude of the subgrid-stress tensor, it is not surprising that the comparison pro- duced results similar to that concerning the SGS stress tensor $\tau_{i j}^{*}$. Thus, global correlations for the subgrid energy transfer field also were calculated to be $\rho \sim 0.85$. In addition, the six random onedimensional intersections depicted in Figure 13 indicate that the model recovers much of the true spatial structure of the magnitude of the flow's energytransfer process as well.

\section{Extension to a Multifractal Model for Scalar Transport.}

Prior studies have indicated that the scalar-energy dissipation rate field associated with high-Reynolds number turbulence,

$$
\chi \equiv-D \nabla \zeta \cdot \nabla \zeta(\mathbf{x}, t)
$$

exhibits multifractal scale-similarity within the inertial scales of the flow. (Prasad \& Sreenivasan 1990, Frederiksen et al 1997). Therefore, just as the multifractal character of the subgrid enstrophy field has been used in Section 4 to derive a model for the subgrid-velocity field, a similar multifractal model can be derived for a dynamically-passive conservedscalar field $\zeta(\mathbf{x}, t)$ in turbulent flow.

For large-eddy simulation, the filtered scalartransport equation, without source term, is given by

$$
\frac{\partial \bar{\zeta}}{\partial t}+\bar{u}_{j} \frac{\partial \bar{\zeta}}{\partial x_{j}}-D \frac{\partial^{2} \bar{\zeta}}{\partial x_{j} \partial x_{j}}=-\frac{\partial}{\partial x_{j}} \underbrace{\overline{u_{j} \zeta}-\bar{u}_{j} \bar{\zeta}}_{\sigma_{j}} .
$$

The non-linear term in $\sigma_{j}$ can be decomposed, giving

$$
\sigma_{j}=\overline{\bar{u}_{j} \bar{\zeta}}+\overline{\bar{u}_{j} \zeta^{s g s}}+\overline{u_{j}^{s g s} \bar{\zeta}}+\overline{u_{j}^{s g s} \zeta^{s g s}}-\bar{u}_{j} \bar{\zeta}
$$

We can use our multifractal model from Section 4 to represent the subgrid velocity components $u_{j}^{s g s}$ appearing in (46) as

$$
u_{j}^{s g s}=\left|\boldsymbol{u}^{\Delta}\right| \frac{\left|\boldsymbol{\omega}^{s g s}\right|}{\left|\boldsymbol{\omega}^{\Delta}\right|} e_{j}^{\Delta} .
$$

A model for the subgrid scalar concentrations $\zeta^{s g s}$ in (46) then can be derived following an approach that parallels the derivation of the subgrid-velocity field in Section 4. First, the subgrid scalar concentrations $\zeta^{s g s}$ can be expressed as an integral over the subgrid scalar-gradient field, using a Green's function approach, as

$$
\zeta^{s g s}=\nabla \cdot \frac{1}{4 \pi} \int_{\mathbf{x}^{\prime}}|\nabla \zeta|^{s g s} \hat{\mathbf{e}}_{\nabla \zeta}^{s g s} \frac{1}{\left|\mathbf{x}-\mathbf{x}^{\prime}\right|} d \mathbf{x}^{\prime} .
$$

This integral is the scalar-field analogue to the BiotSavart integral representation of the subgrid-velocity field. 
The subgrid scalar-gradient magnitude $|\nabla \zeta|^{\text {sgs }}$ term appearing in (48) can now be expressed using multifractal concepts. Because the scalardissipation field $\chi$ exhibits multifractal scalesimilarity in the inertial range of turbulent flow, it possesses a distinct distribution of multifractal multipliers $\mathcal{P}\left(\mathcal{M}_{\varepsilon}\right)$ governing its spatial distribution within the subgrid field. A complete description of that spatial distribution takes the form

$$
|\nabla \zeta|^{s g s} \equiv\left[\chi^{s g s} \prod_{i=1}^{\Delta / \lambda_{\nu}}\left(\mathcal{M}_{\varepsilon}\right)_{i}\right]^{1 / 2}
$$

where $\chi^{s g s}$ is the total amount of scalar dissipation that exists within the subgrid scales. This value can be determined through a Kolmogorov analysis that parallels the determination of the total subgrid enstrophy in Section 4. We know that under $K 41$ theory the scalar-dissipation spectrum $\chi(k)$ exhibits power-law scaling in the inertial range of high Reynolds-number turbulence as

$$
\chi(k) \sim k^{1} .
$$

Thus the total amount of subgrid scalar dissipation can be determined by integrating the scalardissipation spectrum from the smallest-resolved scale $k_{\Delta}$, to the viscous scale $k_{\nu}$, giving

$$
\chi^{s g s}=\frac{\chi^{\Delta}}{2}\left[\left(\frac{k_{\nu}}{k_{\Delta}}\right)^{2}-1\right] .
$$

This can be substituted in (49) to obtain an expression for the spatial distribution of scalar-gradient field magnitude $|\nabla \zeta|^{\text {sgs }}$, which in turn appears in the integral expression for the subgrid scalar field $\zeta^{\text {sgs }}$ in (48). That integral representation can then be evaluated using the central-limit theorem in a fashion closely paralleling the analysis of the Biot-Savart law in Section 4. In the high Reynolds number limit, what emerges is a model for the subgrid scalarconcentration field, where

$$
\zeta^{s g s}=\zeta^{\Delta} \frac{|\nabla \zeta|^{s g s}}{|\nabla \zeta|^{\Delta}}
$$

a form involving only a simple algebraic expression based on quantities available from the resolved scales of the LES calculation.

\section{Concluding Remarks.}

The preceding analysis sets forth a new subgridscale model for the large-eddy simulation of turbulent flow based on the multifractal structure of the subgrid vorticity field. Using a multifractal cascade to represent the distribution of vorticity magnitudes and an additive cascade to represent the distribution of vorticity orientations in the subgrid field, the subgrid velocity components appearing in the subgridstress tensor decomposition can then be expressed as Biot-Savart integrals over the subgrid-vorticity field. The integrals can be simplified using central-limit concepts, and in the high Reynolds-number limit, the subgrid velocity field takes a form requiring only the evaluation of a simple algebraic expression based on the resolved scales of the flow. Using a parallel analysis, the paper also derives a multifractal model for the conserved scalar-transport equation, as a straightforward extension of the same multifractal concepts.

This analysis can be further extended to derive a multifractal model for the Reynolds stresses in the Reynolds Averaged Navier-Stokes (RANS) equation. Using the Taylor Hypothesis, we can treat the time-averages in the RANS equation as spatial averages of quantities advecting past a point at the rate of the mean flow. One can then treat these spatial averages in terms of the multifractal properties outlined above. This would effectively replace the gradient-transport hypothesis as well as associated eddy-viscosity assumptions of current RANS modeling.

\section{Acknowledgments}

The authors gratefully acknouledge the financial support provided by the François-Xavier Bagnoud Foundation, Sion, Switzerland. The DNS data for this study were generously provided by the Center for Turbulence Research at Stanford University and the NASA Ames Research Center, Moffet Field, California.

\section{References}

${ }^{1}$ E. Aurell, U. Frisch, J. Lutsko and M. Vergassola. On the multifractal properties of the energy dissipation derived from turbulence data. J. Fluid Mech., 238:467-486, 1992.

${ }^{2}$ J. Bardina, J.H. Ferziger and R.S. Rogallo. Improved subgrid models for large eddy simulation. AIAA, Paper 801357,1980 .

${ }^{3}$ J.G. Brasseur, and C.H. Wei. Interscale dynamics and local isotropy in high Reynolds number turbulence within triadic interactions. Phys. Fluids 6:842-870, 1994.

${ }^{4}$ J.P. Bouchaud, M. Mézard, and G. Parisi. Scaling and intermittency in Burgers turbulence. Phys. Rev. E, 52:3656$3674,1995$.

${ }^{5} \mathrm{~A}$. Chekhlov, and V. Yakhot. Kolmogorov turbulence in a random-force-driven Burgers equation. Phys. Rev. E, 51:R2739-R2742, 1995.

${ }^{6}$ A.B. Chhabra, and K.R. Sreenivasan. Scale-invariant multiplier distributions in turbulence. Phys. Rev. Lett., 68:2762-2765, 1992.

${ }^{7}$ J.A. Domaradzki, and E. M. Saiki. A subgrid-scale model based on the estimation of unresolved scales of turbulence. Phys. Fluids, 9:2148-2164, 1997.

${ }^{8} \mathrm{~J} . \mathrm{A}$. Domaradzki, and K. Loh. The subgrid-scale estimation model in the physical space representation. Phys. Fluids, 11:2330-2342, 1999.

${ }^{9}$ K. Falconer Fractal Geometry: Mathematical Foundations and Applications. John Wiley \& Sons, 1990. 
${ }^{10} \mathrm{M}$. Farge, K. Schneider, and N. Kevlahan. Non-gaussianity and coherent vortex simulation for twodimensional turbulence using an adaptive orthogonal wavelet basis. Phys. Fluids, 11:2187-2201, 1999.

${ }^{11}$ R.D. Frederiksen, W.J.A. Dahm, and D.R. Dowling. Experimental assessment of fractal scale similarity in turbulent flows. Part 1. One-dimensional intersections. J. Fluid Mech., 327:35-72, 1996.

${ }^{12}$ R.D. Frederiksen, W.J.A. Dahm, and D.R. Dowling. Experimental assessment of fractal scale similarity in turbulent flows. Part 3. Multifractal scaling. J. Fluid Mech., 338:127-155, 1997.

${ }^{13}$ U. Frisch. Turbulence: The Legacy of A.N. Kolmogorov. Cambridge University Press, 1995.

${ }^{14} \mathrm{M}$. Germano. A proposal for a redefinition of the turbulent stresses in the filtered Navier-Stokes equations. Phys. Fluids, 29:2323-2324, 1986.

${ }^{15}$ M. Germano, U. Piomelli, P. Moin, and W.H. Cabot. A dynamic subgrid-scale eddy viscosity model. Phys. Fluids, A3:1760-1765, 1991.

${ }^{16} \mathrm{~T}$. Gotoh, and R. Kraichnan. Statistics of decaying Burgers turbulence. Phys. Fluids A, 5:445-457, 1993.

${ }^{17} \mathrm{~B}$.J. Geurts Inverse modeling for large-eddy simulation. Phys. Fluids, 9:3585-3587, 1997.

${ }^{18} \mathrm{~J}$. Jimenez, A.A. Wray, P.G. Saffman, and R.S. Rogallo The Structure of Intense Vorticity in Isotropic Turbulence. $J$. Fluid Mech., 255:65-90, 1993.

${ }^{19}$ R.M. Kerr, J.A. Domaradzki, and G. Barbier. Smallscale properties of nonlinear interactions and subgrid-scale energy transfer in isotropic turbulence. Phys. Fluids, 8:197208,1996 .

${ }^{20} \mathrm{R}$. Kraichnan. Models of intermittency in hydrodynamic turbulence. Phys. Rev. Lett., 65:575-578, 1990.

${ }^{21} \mathrm{~S}$. Liu, C. Meneveau, and J. Katz. On the properties of similarity subgrid-scale models as deduced from measurements in a turbulent jet. J. Fluid Mech., 275:83-119, 1994.

$22 \mathrm{~J}$. Mansfield, O. Knio, and C. Meneveau. A dynamic LES scheme for the vorticity transport equation: formulation and a priori tests. J. Comp. Phys., 145:693-730, 1998.

${ }^{23}$ C. Meneveau, and K.R. Sreenivasan. Simple multifractal cascade model for fully developed turbulence. Phys. Rev. Lett., 59:1424-1427 (1987).

${ }^{24} \mathrm{C}$. Meneveau, and K.R. Sreenivasan. The multifractal nature of turbulent energy dissipation. J. Fluid Mech., 224:429-484, 1991.

${ }^{25} \mathrm{C}$. Meneveau. Analysis of turbulence in the orthonormal wavelet representation. J. Fluid Mech., 232:469-520, 1991.

${ }^{26} \mathrm{C}$. Meneveau, T. Lund, and W. Cabot. A Lagrangian dynamic subgrid-scale model of turbulence. J. Fluid Mech., 319:353-385, 1996.

${ }^{27} \mathrm{O}$. Metais, and M. Lesieur. Spectral large-eddy simulation of isotropic and stably stratified turbulence. J. Fluid Mech., 239:157-194, 1992.

${ }^{28}$ A. Misra, and D.I. Pullin. A vortex-based subgrid stress model for large eddy simulation. Phys. Fluids, 9:2443-2454, 1997.

${ }^{29} \mathrm{H}$. Peitgen, H. Jurgen, and D. Saupe. Chaos and Fractals: New Frontiers of Science. Springer-Verlag (1992)

${ }^{30}$ R.R. Prasad, C. Meneveau, and K.R. Sreenivasan. The multifractal nature of the dissipation field of passive scalars in fully turbulent flows. Phys. Rev. Lett., 61:74-77 (1988)

${ }^{31}$ R.R. Prasad, and K.R. Sreenivasan. Quantitative threedimensional imaging and the structure of passive scalar fields in fully turbulent flows. J. Fluid Mech., 216:1-34 (1990)

${ }^{32}$ D.I. Pullin, and P.G. Saffman. Reynolds stresses and one-dimensional spectra for a vortex model of homogeneous anisotropic turbulence. Phys. Fluids, 6:1787, 1994.

${ }^{33}$ P. Sagaut. Large-Eddy Simulation for Incompressible Flow. Springer-Verlag (2000)
${ }^{34} \mathrm{~A}$. Scotti, and C. Meneveau. Fractal model for coarsegrained nonlinear partial differential equations. Phys. Rev. Lett., 78:867-870, 1997.

${ }^{35} \mathrm{~A}$. Scotti, and C. Meneveau. A fractal model for large eddy simulation of turbulent flow. Physica D, 127:198-232, 1999.

${ }^{36} \mathrm{~J}$. Smagorinsky. General circulation experiments with the primitive equations: I. The basic equations. Mon. Weather Rev., 91:99-164, 1963.

${ }^{37}$ K.R. Sreenivasan, and R.R. Prasad. New results on the fractal and multifractal structure of the large Schmidt number passive scalars in fully turbulent flows. Physica $D$, 38:322-329 (1989).

${ }^{38}$ K.R. Sreenivasan. Fractals and multifractals in fluid turbulence. Annu. Rev. Fluid. Mech., 23:539-600, 1991.

${ }^{39}$ K.R. Sreenivasan. On local isotropy of passive scalars in turbulent shear flows. Proc. R. Soc. Lond. A, 434:165-182, 1991.

${ }^{40}$ K.R. Sreenivasan, and G. Stolovitzky. Turbulent cascades. J. Stat. Phys., 78:311-333, (1995).

${ }^{41}$ S. Stoltz, and N.A. Adams An approximate deconvolution procedure for large-eddy simulation. Phys. Fluids. 11:1699-1701, 1999.

${ }^{42}$ Y. Zang, R.L. Street, and J. Koseff. A dynamic mixed subgrid-scale model and its application to turbulent recirculating flows. Phys. Fluids, A5:3186-3196, 1993. 

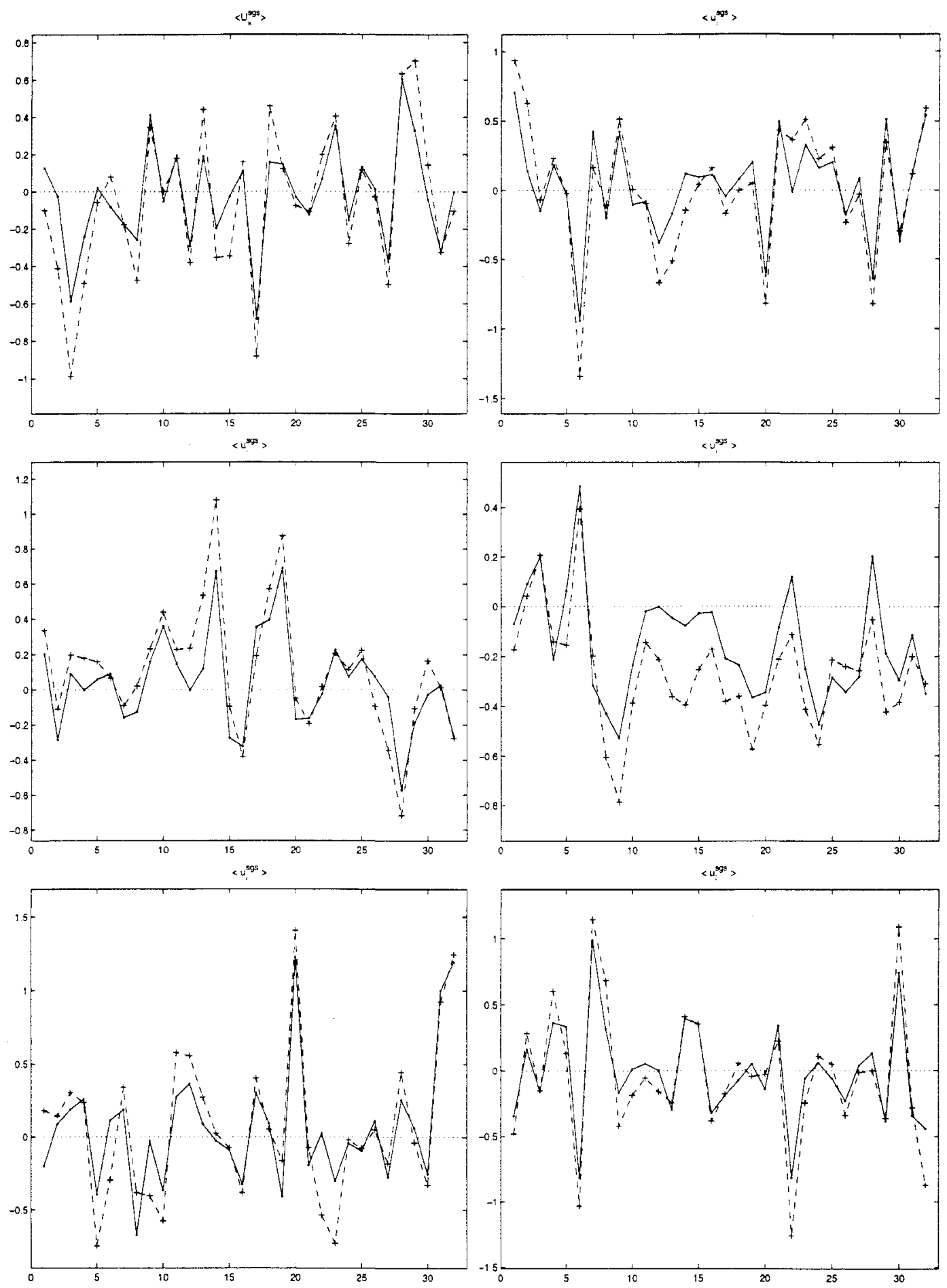

Fig. 11 Filtered Subgrid-Velocity Components. Six randomly-selected one-dimensional intersections from the filtered subgrid-velocity component field, $\overline{u_{i}^{s g s}}$, with DNS values (solid) vs. Model values (dashed). The comparison provides a basic test of the present model for the subgrid velocity field. Global correlations with the DNS data are calculated to be $\rho \sim 0.91$. Note that the model does well at recovering the velocity magnitudes as well. 

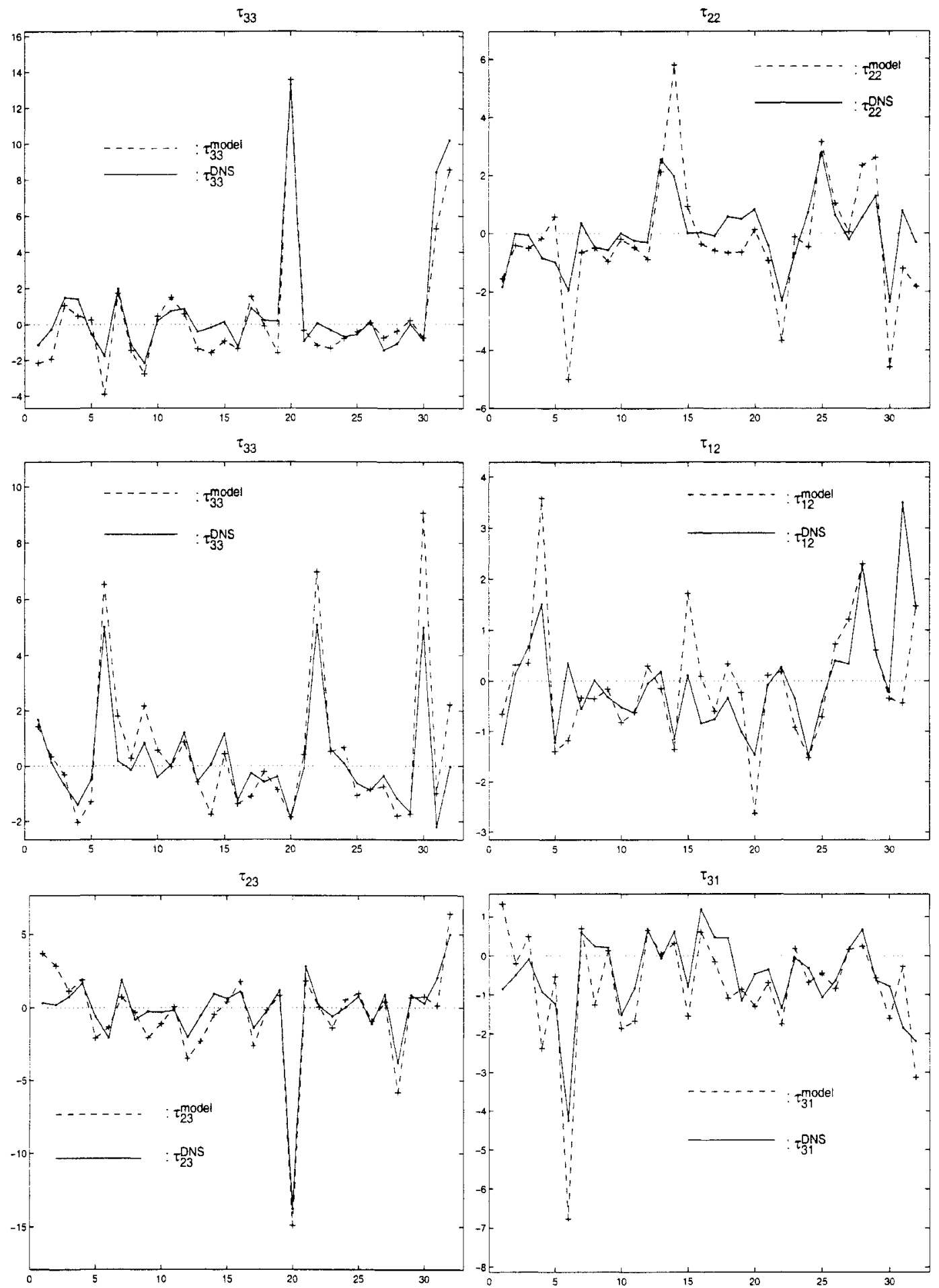

Fig. 12 Subgrid-Stress Tensor Components, $\tau_{i j}^{*}$. Six randomly-selected one-dimensional intersections from the subgrid-stress tensor component field. $\tau_{i j}^{*}$ is comprised exclusively of those terms from (4) containing contributions from the modeled subgrid field. DNS values are shown (solid) vs. model values (dashed). Global correlations between the two data sets average $\rho \sim 0.85$. Note good magnitude as well as phase agreement throughout. 

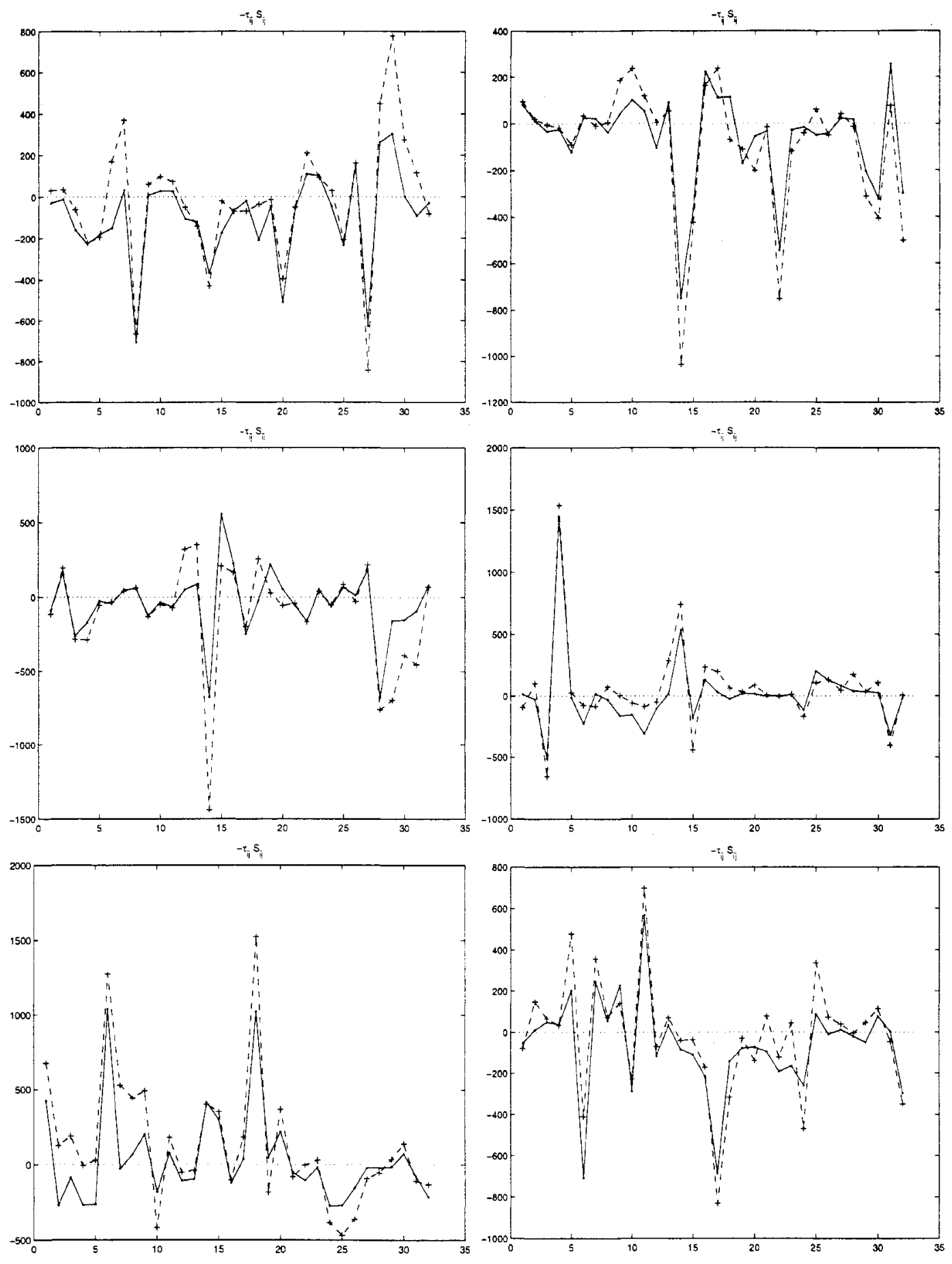

Fig. 13 Subgrid-Energy Production Field, $\mathcal{P}^{*} \equiv-\tau_{i j}^{*} \bar{S}_{i j}$. Six randomly-selected one-dimensional intersections from the subgrid-energy production field, with DNS values (solid) vs. model values (dashed). $\mathcal{P}^{*}$ contains only the subgrid-energy production due to the effects of the subgrid contributions to the stress tensor $\tau_{i j}$. Global correlations between the two data sets are $\rho \sim 0.85$. The modeled energy-production field captures much of the detail of the true SGS energy production field, in both orientation and magnitude. 